\title{
Neonatal screening for hereditary fructose intolerance: frequency of the most common mutant aldolase B allele (A149P) in the British population
}

\author{
C L James, P Rellos, M Ali, A F Heeley, T M Cox
}

\begin{abstract}
Hereditary fructose intolerance (HFI) causes severe and sometimes fatal metabolic disturbances in infants and children but responds to dietary treatment. To determine the practicability of screening newborn infants for HFI, we have investigated the frequency of the most common and widespread mutant allele of aldolase B, A149P, in the neonatal population. The polymerase chain reaction was used to amplify aldolase $B$ exon 5 genomic sequences in DNA present in dried blood specimens preserved on Guthrie cards. The A149P mutation was identified by discriminatory hybridisation to allele specific oligonucleotides and confirmed independently by digestion with the restriction endonuclease BsaHI. Twenty-seven A149P heterozygotes were identified by the molecular analysis of aldolase $B$ genes in blood samples obtained from a random cohort of 2050 subjects born in 1994 and $1995,1.32 \pm 0.49 \%$ (95\% confidence level). Although no A149P homozygotes were identified, the data allow the frequency of 1 in 23000 homozygotes for this allele to be predicted. Our findings have implications for establishing an interventional mass screening programme to identify newborn infants with HFI in the UK.

(f Med Genet 1996;33:837-841)
\end{abstract}

Key words: aldolase B; fructose intolerance; neonatal population; genetic screening.

Department of

Medicine, University

of Cambridge,

Addenbrooke's

Hospital, Hills Road,

Cambridge CB2 2QQ

UK

C L James

P Rellos

$\mathrm{M}$ Ali

T M Cox

Regional Biochemical Genetics Screening Unit, Peterborough District Hospital,

Thorpe Road

Peterborough PE3

6DA, UK

A F Heeley

Correspondence to:

Professor Cox.

Received 25 April 1996 Revised version accepted fo publication 30 May 1996

disease that results from a deficiency of aldolase B (fructose-bisphosphate aldolase, EC 4.1.2.13) in the liver, intestine, and kidney.

First manifest at weaning, HFI causes vomiting, abdominal pain, hypoglycaemic attacks, and growth retardation; continued ingestion of fructose and sucrose leads to renal disease and cirrhosis. ${ }^{3}$ Symptomatic HFI resolves with the institution of a strict exclusion diet and with prompt treatment in infancy, permanent visceral injury can be avoided. Until recently, the mainstay of diagnosis has been the intravenous fructose tolerance test or enzymatic analysis of intestinal or liver biopsy specimens, but these methods are unsuitable for diagnosis in severely affected infants. Latterly, we have characterised many mutations in the aldolase $\mathrm{B}$ gene that cause $\mathrm{HFI}^{4}$ and the first to be identified (the missense mutation $\mathrm{A} 149 \mathrm{P}^{5}$ ) accounts for $>80 \%$ of disease alleles in the United Kingdom. $^{4-6}$ Thus, molecular analysis of the human aldolase B gene obtained from genomic DNA has particular value for the diagnosis and carrier detection of HFI.

If identified and treated before permanent injury occurs, hereditary fructose intolerance is compatible with a normal quality and duration of life. There are thus compelling reasons to consider the introduction of genetic testing for the disease during the presymptomatic neonatal period. To determine the practicability of this preventative strategy, a robust estimate of the gene frequency in the target population is needed. This would determine the incidence of the disease and permit an accurate assessment of the extent to which the benefits of its early detection would outweigh the cost of a neonatal screening programme.

To estimate the incidence of HFI in the population, we have taken advantage of (1) the predominance of the mutant A149P allele in British patients known to be suffering from the disease, and (2) the quadratic relationship, based on the Hardy-Weinberg equilibrium, between the frequencies of heterozygotes and homozygotes for rare autosomal recessive disorders. ${ }^{7}$ Accordingly, the polymerase chain reaction was used to amplify aldolase $B$ gene sequences present in archival DNA obtained from blood samples taken at birth for the routine detection of phenylketonuria or hypothyroidism. Molecular analysis of the gene for the A149P mutation was carried out by discriminatory hybridisation to allele specific oligonucleotides and independently confirmed by digestion with the restriction endonuclease BsaHI.
Methods

DNA SAMPLES

Samples of genomic DNA were extracted for molecular analysis of the aldolase B gene from dried blood specimens that were preserved on Guthrie screening cards after use in the East Anglian regional biochemical genetic screening unit. Ethical permission for the conduct of these studies anonymously was granted from the ethics committee of Addenbrooke's Hospital, Cambridge, which regulates human experi- 
mentation carried out in the research laboratory. Mutation analysis was performed using catalogued blood samples, whose number was recorded, in the event that homozygotes for aldolase B mutations (who would have HFI) were detected; such patients could then be identified independently after decoding the archive. Molecular analysis of the aldolase B gene was carried out using samples of template DNA obtained from blood spots that had already been used for routine biochemical screening to detect phenylketonuria and hypothyroidism within the first week of life. The archive contains dried blood specimens that remain after completion of biochemical assays in 25000 newborn infants each year; for the conduct of the present study, 2200 samples obtained in batches consecutively from week 43 of 1994 to week 13 of 1995 were analysed (to minimise the chance of successive studies of full or half sibs).

EXTRACTION OF GENOMIC DNA AND MOLECULAR ANALYSIS OF ALDOLASE B GENES

Circles $\left(1 \mathrm{~mm}^{2}\right)$ were punched out of the dried blood spots on Guthrie cards with a hollow metal punch and placed in polypropylene tubes (Eppendorf Ltd). Twenty microlitres of $0.01 \mathrm{~mol} / 1 \mathrm{Na0H}$ were added and the tubes were raised to $95^{\circ} \mathrm{C}$ on a heating block for 10 minutes. After cooling, the mixture was neutralised by addition of $5 \mu \mathrm{l}$ of $0.04 \mathrm{~mol} / 1$ $\mathrm{HCl}$.

To amplify exon 5 sequences of the aldolase $B$ - gene, the polymerase chain reaction (PCR) was established by addition of $75 \mu$ l of $1 \times$ Promega Taq buffer containing $\mathrm{MgCl}_{2}$ (2 $\mathrm{mmol} / \mathrm{l})$ oligonucleotide primers $(0.2 \mathrm{mmol} / \mathrm{l})$, $1 \mathrm{mmol} / 1$ each of the four dNTPs, and 2 units of Taq polymerase (Promega). The sequences of the primers were X5: GAG CAC CTG CTG ACA GAT GCT and X $5^{+}$: GAG CGC TGT GCT CAG TAC AAG. These permit the amplification of a $150 \mathrm{bp}$ product encompassing codons 137-174 of the aldolase $B$ gene with some additional intron sequence. Forty cycles of PCR were carried out under mineral oil with a one minute denaturing step at $94^{\circ} \mathrm{C}$, annealing for 30 seconds at $50^{\circ} \mathrm{C}$, followed by amplification at $72^{\circ} \mathrm{C}$ for one minute.

After removing $10 \mu \mathrm{l}$ of the reaction mixture for agarose gel electrophoresis and staining with ethidium bromide to confirm satisfactory amplification, the remainder was separated from solid debris and transferred to a separate tube to which $20 \mu \mathrm{l}$ of $100 \mathrm{mmol} / 1$ Tris $\mathrm{HCl} / 1$ $\mathrm{mmol} / 1$ EDTA pH 8.0 was added. Fifty microlitres of phenol/chloroform/isoamyl alcohol (25:24:1 by volume) were added and the mixture was agitated vigorously and then centrifuged at $12000 \mathrm{~g}$ for two minutes. Ninety microlitres of the aqueous layer was removed and $10 \mu \mathrm{l}$ of $3 \mathrm{~mol} / \mathrm{l}$ sodium acetate and $250 \mu \mathrm{l}$ of $100 \%$ ethanol was added to precipitate the DNA and the mix was kept at $-20^{\circ} \mathrm{C}$ for at least 16 hours. After centrifuging for $12000 \mathrm{~g}$ for 15 minutes, the fluid phase was removed carefully and the DNA precipitate was carefully dried in air before being dissolved in $10 \mu$ lof distilled $\mathrm{H}_{2} \mathrm{O}$. The products were heated to $95^{\circ} \mathrm{C}$ for three minutes, $10 \mu \mathrm{l}$ of $20 \times$ SSC buffer was added, and the DNA solution was then blotted onto a Hybond $\mathrm{N}$ nylon membrane using a gridded blotting apparatus (Gibco BRL), hybridised with the ${ }^{32} \mathrm{P}$-end labelled A149P mutation specific oligonucleotide and washed at the discriminatory temperature $\left(67.5^{\circ} \mathrm{C}\right)$ before drying and autoradiography, as previously described. ${ }^{6}$

Samples that hybridised positively with the mutant oligonucleotide probe were reamplified and screened independently. Those samples that gave reproducible hybridisation signals were finally amplified independently from fresh genomic DNA templates and the presence of the A149P mutation was confirmed by digestion with the restriction endonuclease $B s a \mathrm{HI}$, an isoschizomer of AhaII. ${ }^{5}$

\section{Results}

Of the 2200 blood samples obtained from Guthrie cards, DNA amplification products suitable for aldolase B genotyping were obtained from 2050 . Our inability to obtain a single product from the template DNA in less than $5 \%$ of the archival samples was not further investigated, but potential causes would include DNA degradation with or without microbial contamination and the inhibitory effects of adherent proteins, including oxidised haemoglobin. The possibility that errors in the neutralisation of $\mathrm{NaOH}$ used to elute the template occurred is largely excluded by the failure to obtain amplification products in a fresh PCR conducted with the original refractory sample in most cases.

During the initial screening of these amplification products by dot blot filter hybridisation to the mutant A149P oligonucleotide, approximately 200 samples were considered for further analysis. To ensure accuracy and avoid bias, a further 200 blood samples, whose amplification products were considered not to hybridise with the A149P oligonucleotide probe at the discriminatory temperature, were also reamplified in the PCR and subjected to further independent genotyping.

Twenty-seven blood spot samples from the initial screening of those that were considered to hybridise positively or to give putative hybridisation signals with the A149P oligonucleotide were again amplified for analysis by restriction endonuclease digestion (fig 1 ). This analysis was also conducted on the batch of 200 individual products obtained from samples that were wild type by hybridisation analysis. Digestion of all 27 products with $B s a \mathrm{HI}$ confirmed the presence of one copy of the A149P allele. Identical analysis of the independent batch of 200 PCR products obtained from blood samples considered to be homozygous wild type (A149+/A149 ${ }^{+}$) by allele specific hybridisation confirmed this genotype in all cases. Finally, the heterozygous genotype A149P/A149 ${ }^{+}$was verified independently by restriction endonuclease digestion of freshly amplified DNA, using appropriate controls, in all 27 samples. No homozygous A149P samples were identified in the cohort. Since the occurrence of 27 positive observations occur- 
A

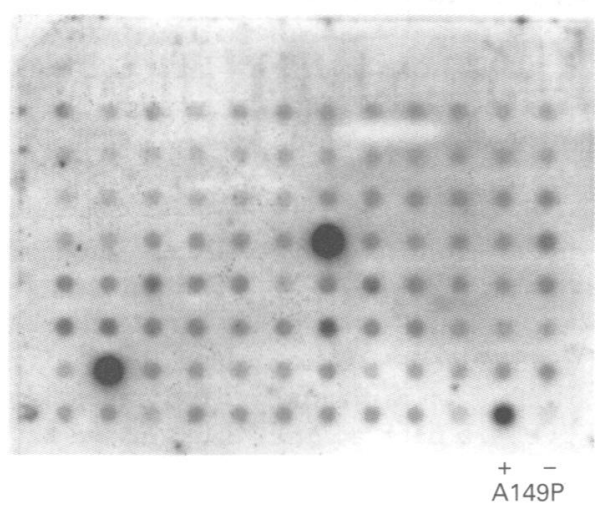

B

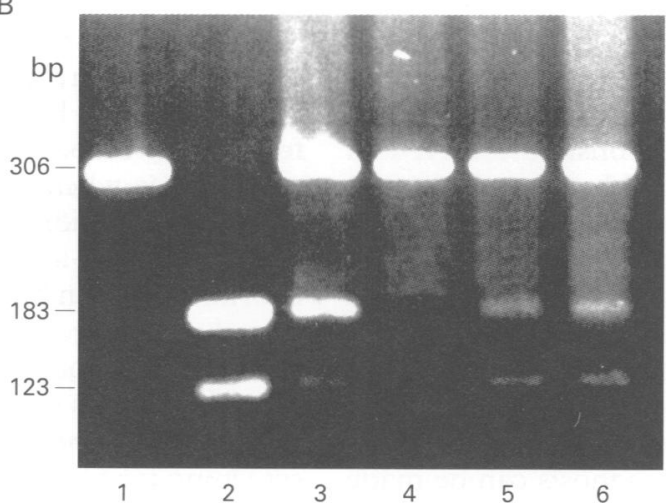

Figure 1 (A) Allele specific analysis of amplified DNA from Guthrie blood spots. Dot blot hybridisation of 96 well gridded filter with mutant A149P allele specific

oligonucleotide probe after washing under discriminatory conditions. Plasmid DNA controls with wild type and mutant aldolase $B$ sequences are depicted as shown in the lower right hand grid spots. (B) Confirmatory restriction endonuclease digestion for the A149P allele. Agarose gel electrophoresis of $B s a H I$ digests of aldolase $B$ exon 5 sequences amplified independently in the PCR from

genomic DNA in further dried blood samples obtained from Guthrie cards. ${ }^{5}$ The undigested exon 5 fragment of $306 \mathrm{bp}$ is cleared into two fragments of 183 and $123 \mathrm{bp}$ when it harbours the A149P mutation. Track 1, wild type control $D N A$; track 2 , homozygous $A 149 P$ control DNA; track 3 , heterozygous $A 149 P$ control DNA; track $4, D N A$ amplified from blood sample 218, negative for $A 149 P$ by allele specific hybridisation (see ( $A$ ) above); track 5, DNA from blood sample 168, positive for A149P by hybridisation (see (A) above); track 6, DNA from blood sample 288, also positive for A149P by allele specific hybridisation, as shown in $(A)$.

ring independently in a sample size of more than 2000 is in effect a sporadic event, the confidence limits of this frequency would be described by the Poisson distribution. ${ }^{8}$ The standard error of this distribution is given by the square root of its mean frequency. Thus, the $95 \%$ confidence limits of the observed heterozygote frequency would be $100 \times(27 \pm$ $1.96 \sqrt{ } 27) / 2050=1.317 \pm 0.49 \%$. The corresponding gene frequency of A149P would be $100 \times(27 \pm 1.96 \sqrt{ } 27) / 4100=0.659 \pm$ $0.248 \%$

\section{Discussion}

As a step towards the introduction of neonatal screening for hereditary fructose intolerance, we have determined the frequency of the most common mutant allele responsible for this disorder in the British population. The observed gene frequency (q) $6.59 \pm 2.48 \times 10^{-3}$ corresponds to a heterozygote frequency of $1.32 \pm 0.49 \%$ in an unselected cohort of new- born infants in the UK. The predicted incidence of HFI ascribable to this allele can be calculated from the Hardy-Weinberg equilibrium as $1 / q^{2}=1$ in 23000 births. The $95 \%$ confidence limits would be 1 in 59500 to 1 in 12200 births.

Hereditary fructose intolerance is analogous to the nutritional genetic disorder phenylketonuria, a metabolic condition that, untreated, leads to permanent brain injury and disability. Both phenylketonuria and hereditary fructose intolerance are associated with a presymptomatic neonatal period and in the early stages respond completely to dietary exclusion therapy. ${ }^{239}$ Since the A149P mutation in the human aldolase $\mathbf{B}$ gene is responsible for $\sim 80 \%$ of disease alleles in British patients with HFI, neonatal testing for this disorder, like hyperphenylalanaemia based on biochemical assay, is readily accomplished.

Given the demonstrable value of diagnosing phenylketonuria in the early presymptomatic period after birth, mass neonatal screening by blood spot analysis was introduced for this condition in 1968. The frequency of phenylketonuria varies in different populations: in the London region of England the incidence is reported to be 70 per million ${ }^{9}$ or 1 in 14300 live births. In the East Anglian regional genetic service, a mean frequency of 1 in 17000 has been observed over more than 10 years from a birth total of about 25000 per annum. This indicates that the East Anglian population is broadly representative of the British population. Since the annual live birth rate in England and Wales is $\sim 660000,{ }^{10}$ the regional British population screening programmes for phenylketonurias would, on average, identify 46 new cases of this metabolic disorder. In the light of the current study, 29 cases of hereditary fructose intolerance would be detected by genetic testing for the mutant A149P allele annually (95\% confidence limits 11-54).

Molecular analysis of the aldolase $B$ genes in British patients with hereditary fructose intolerance indicates that the A149P variant accounts for $\sim 80 \%$ of mutant alleles. Thus, the estimated disease frequency of HFI caused by all mutant alleles of aldolase $B$ would be closer to 1 in 18000 live births. This corresponds closely to the sole estimate, reported by Gitzelmann and Baerlocher ${ }^{11}$ from Switzerland, of approximately 1 in 20000 , an estimate based on the diagnosis of five patients with HFI at the Kinderspital Zurich and on 96000 consecutive live births. Using the Poisson distribution, the confidence limits for this estimate are calculated to be $1 / 100000$ $1 / 11000$ and thus too imprecise for predicting the costs or outcomes of a neonatal screening programme in the UK. Moreover, the distribution of mutant alleles of aldolase B in the Swiss population differs significantly from that obtained by the study of HFI patients in the British population. ${ }^{12}$ Consequently, the introduction of a screening programme for HFI using DNA testing of blood samples from newborn Swiss infants would need to include genotyping for the $A 174 \mathrm{D}, \mathrm{N} 334 \mathrm{~K}$, and other rarer widespread alleles that contribute, with A149P, 
significantly to the genetic burden of this disease in non-British patients. ${ }^{46}$

The finding that $1.32 \pm 0.49 \%$ of subjects in the neonatal population under study carry the A149P allele of aldolase B also has significance for the molecular diagnosis of hereditary fructose intolerance by genetic testing of those who suffer sugar intolerance. Homozygosity for A149P is clearly tantamount to a diagnosis of HFI, but it can now be predicted that the finding of a heterozygous A149P + /- genotype would occur in less than $2 \%$ of normal people in any population. Thus, a person with symptoms who requests DNA testing and who is assigned the heterozygous A149P + /- genotype is likely to suffer from HFI. Such people would harbour another mutant aldolase B allele on the other $\left(\mathrm{A} 149^{+}\right)$chromosome. Given that A149P accounts for most but not all of the mutant alleles responsible for HFI in British patients, compound heterozygosity will be expected in an appreciable proportion of those with the disease. Clearly, until all representative mutant aldolase alleles can be identified and readily tested for, A149P $+/-$ heterozygotes with symptoms who request diagnostic genetic tests should thus be considered to have HFI and given appropriate dietary advice until a definitive diagnosis can be made by other means.

The demonstration that the prevalence of the A149P allele of aldolase B approaches polymorphic frequency in the United Kingdom provides an important basis for understanding the evolutionary genetics of hereditary fructose intolerance in Europe. Brooks and $\operatorname{Tolan}^{13}$ have provided evidence that all human chromosomes harbouring the A149P allele in our British patients with HFI and in other subjects were derived from an ancestral chromosome that had undergone a single mutational event. This conclusion is based on the finding of an absolute association between A149P and linked alleles at two silent loci within intron 6 of the aldolase B gene; similar studies of another widespread mutation, A174D prevalent in southern Europe, ${ }^{46}$ showed strong association with allelic counterparts at these silent loci. It therefore seems probable that inactivating mutations in the aldolase B gene which cause hereditary fructose intolerance arose during a prolonged period of minimal selection against this disorder during human evolution, and spread by genetic drift regionally within European populations. ${ }^{412}$ Hereditary fructose intolerance indeed escaped recognition until recent times (it was first reported in 1956), when changing dietary compositions have increased the risk of harmful exposure to noxious sugars in predisposed people. ${ }^{414}$

The outcome of this study, which shows that the frequency of heterozygous carriers of the A149P allele responsible for hereditary fructose intolerance is $1.32 \pm 0.49 \%$, favours the introduction of a genetic screening test in the British population. We would predict that about 30 cases of this treatable disorder could be identified annually in the presymptomatic period, which is comparable to the number of cases of classical phenylketonuria currently detected by analysis of dried blood samples. Since contemporary legislation ensures that these samples are collected during the first week of life for mass population screening by regional health services, the means for experimental evaluation of a programme to prevent disease occurring in those predisposed to fructose intolerance are already in place. It is pertinent to consider the best means by which aldolase $B$ gene mutations can be detected. We have preferred to use the procedure described here because it proved to be robust and, even using dried archival samples, $>95 \%$ amplified satisfactorily for analysis. The amplification refractory mutation system (ARMS), which we have used for detection of mutations at the human glucocerebrosidase locus ${ }^{15}$ and in the human aldolase $\mathrm{B}$ gen $\mathrm{e}^{16}$ using DNA extracted from fresh blood samples, proved to be less satisfactory when applied to archival DNA eluted from oxidised blood spots dried onto cellulose paper, as in this study. Allele specific hybridisation can be carried out using an automated procedure based on 96 well plates and, if suitably mechanised for population screening, labour costs could be minimised. The estimated direct laboratory cost of our blood spot screening for phenylketonuria for 30000 samples annually $(£ 0.75)$ would not then be expected to be greatly exceeded by the automated PCR based procedure described in this paper (reagents $£ 0.60$, labour $£ 0.75$ per sample).

Given the predicted frequency of HFI and the known efficacy of prompt dietary intervention, the introduction of a prospective trial of neonatal screening for HFI by testing DNA in blood spots is now mandatory. Such a trial would assess the value of mass population screening and prompt the development of dietary treatment for this disease in the early neonatal period before natural taste aversions develop. In addition, the results obtained from an appropriately designed and more comprehensive retrospective trial could add greatly to our knowledge of the natural course of hereditary fructose intolerance.

The first two authors contributed equally to the conduct of this study. Mrs J B Grantham kindly provided secretarial assistance. This work was supported by a grant from the Medical Research Council and the Wellcome Trust.

1 Chambers RA, Pratt RTC. Idiosyncracy to fructose. Lancet 1956;ii: 340

2 Gitzelmann R, Steinmann B, Van den Berghe G. Disorders of fructose metabolism. In: Scriver CR, Beaudet A, Sly of fructose metabolism. In: Scriver CR, Beaudet A, Sly WS, Valle D, eds. The metabolic and molecular basis of inher-
ited disease. 7th ed. New York: McGraw-Hill, 1995:905-34. Odievre M, Gentil C, Gautier C, et al. Hereditary fructose Odievre $M$, Gentil C, Gautier C, et al. Hereditary fructose intolerance in childhood. Diagnosis, management and

course in 55 patients. $A m$ f Cox TM. Aldolase

1994;8:62-71.
Cross NCP, Tolan DR, Cox TM. Catalytic deficiency of

5 Cross NCP, Tolan DR, Cox TM. Catalytic deficiency of
human aldolase $\mathrm{B}$ in hereditary fructose intolerance human aldolase B in hereditary fructose intolerance caused by a common missense mutation. Cell 1988; 53:881-5.

6 Cross NCP, Cox TM. Molecular analysis of aldolase B genes in the diagnosis of hereditary fructose intolerance in the United Kingdom. Q f Med 1989;73:1015-20.

7 Hardy GH. Mendelian proportions in a mixed population. Science 1908;28:49-50.

8 Snedecor GW, Cochran WG. Statistical methods. 7th ed. Ames: Iowa State University Press, 1980:130-4.

9 Scriver CS, Kaufman S, Eisensmith RC, Woo SLC. The hyperphenlalaninemias. In: Scriver CR, Beaudet A, Sly WS hyperphenylalaninemias. In. Scriver CR, Beaudet A, Sly ease. 7th ed. New York: McGraw-Hill, 1995:1015-75. 
10 Census. Births and Deaths in England and Wales 1988.London: Her Majesty's Stationery Office, 1988

11 Gitzelman Fructose in der Nahrung. Pädiatr Fortbildk Praxis 1973;

12 Cross NCP, de Franchis R, Sebastio G, et al. Molecular analysis of aldolase $B$ genes in hereditary fructose intolerance. Lancet 1990;335:306-9.

13 Brooks CC, Tolan DR. Association of the widespread A149P hereditary fructose intolerance mutation with newly-identified sequence polymorphisms in the aldolase B gene. Am f Hum Genet 1993;52:835-40.

14 Deerr N. History of sugar. Vols 1 and 2. London: Chapman and Hall, 1949.

15 Mistry PK, Smith SJ, Ali M, Hatton CSR, McIntyre N, Cox TM. Genetic diagnosis of Gaucher's disease. Lancet 1992; 339:889-92.

16 Ali M, Rosien U, Cox TM. DNA diagnosis of fatal fructose intolerance from archival tissue. $Q \mathcal{F} \mathrm{Med}$ 1993;86:25-30. 